None of the proposed cooperative explanations for synchrony ${ }^{1}$ seem to apply to waving in $U$. annulipes. First, there are no predators who would be confused by group synchrony thereby reducing each male's predation risk. Second, synchrony does not increase group conspicuousness to distant females because males wave synchronously only when a female is $<10 \mathrm{~cm}$ away (P. B., unpublished data). The most plausible explanation is one recently proposed for synchronous chorusing by a katydid ${ }^{2}$. Modelling shows that competition between males to call before their neighbours can lead to synchrony. Males compete to call first because females prefer leading calls.

This so-called 'precedence effect', whereby signal receivers show greater responsiveness to the earlier signal in a pair, is found in many acoustic situations, including sound localization in humans. It has not, however, been reported in a visual communication system. In U. annulipes, the visited male produced leading waves significantly more often than his neighbours $(4.5 \pm 3.5$ compared with $3.2 \pm 3.3$ waves; Wilcoxon test, $n=45, P<0.02)$. Female $U$. annulipes may prefer leading signals and synchrony may arise as an incidental effect of competition between males to signal first.

This is the first example of synchronous production of a non-bioluminescent visual signal. Mechanisms of visual signal perception and processing must possess the properties that were previously thought to limit synchronized courtship signalling to acoustic and bioluminescent channels ${ }^{1}$.

Patricia Backwell, Michael Jennions

Neville Passmore

Department of Zoology,

University of the Witwatersrand,

Braamfontein, Johannesburg 2050, South Africa

e-mail:backwelp@naos.si.edu

John Christy

Smithsonian Tropical Research Institute,

Apartado 2072, Balboa, Republic of Panama

1. Greenfield, M. D. Annu. Rev. Ecol. Syst. 25, 97-126 (1994).

2. Greenfield, M. D. \& Roizen, I. Nature 364, 618-620 (1993).

3. Sismondo, E. Science 249, 55-58 (1990).

4. Backwell, P. R. Y. \& Passmore, N. I. Behav. Ecol. Sociobiol. 38, 407-416 (1996).

5. Gordon, H. R. S. An. Behav. 134, 238-241 (1958).

\section{Connexin mutations and hearing loss}

Kelsell et al. ${ }^{1}$ provide convincing evidence that mutations in the gene encoding the gap-junction protein connexin 26 (Cx26) are responsible for autosomal recessive non-syndromic hearing loss at the DFNB1 locus on chromosome 13q12. They also report a small family with apparent autosomal dominant congenital hearing loss and autosomal dominant palmoplantar kerato-

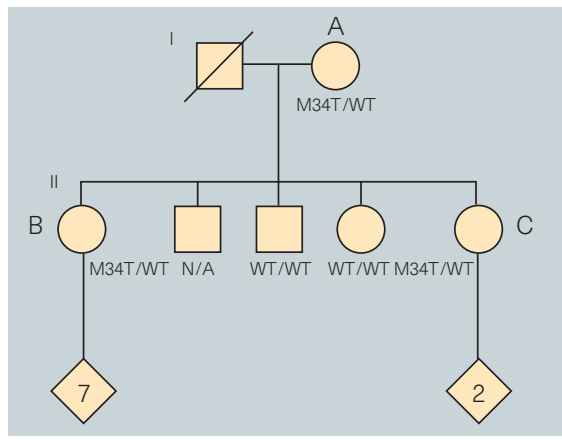

Figure 1 Pedigree structure in a family with the M34T variant of Cx26. Individual A (I:2) is 83 years old, individual B (II:1) is 57 years old and individual C (Il:5) is 55 years old. The numbers in diamonds represent numbers of children. WT, wild-type; N/A, not available.

derma (PPK) in which two siblings with profound hearing loss are heterozygous for a single base-pair substitution resulting in a methionine-to-threonine (ATG-to-ACG) change in codon 34 (M34T) of Cx26 (refs $1,2)$. The authors conclude that the M34T change is the genetic basis of profound hearing loss in this family and suggest, on this basis, that the Cx26 gene is responsible for autosomal dominant non-syndromic hearing loss (ADNSHL) at the DFNA3 locus chromosome 13q12 (ref. 3). We have identified a family in which the M34T variant is not associated with hearing loss, suggesting that this conclusion might be premature.

In a Cx26 mutation screen of 100 random individuals from the midwestern United States, we discovered one person who carries the M34T allele. DNA sequencing confirmed this person and two other family members to be heterozygous for this mutation. All three individuals have excellent hearing (Figs 1, 2) ${ }^{4}$. Individuals B and C report that their children, the youngest of whom is 24 , all have normal hearing.

There are various explanations for these data. First, the M34T variant might be responsible for a form of ADNSHL that is not expressed in certain individuals. But the identification of multiple heterozygous individuals with no evidence of inherited hearing loss argues against this suggestion. Second, individuals in this family might carry a compensatory change that nullifies the effects of the M34T variant. However, single-stranded conformational polymorphism (SSCP) and sequence analysis of the $\mathrm{Cx} 26$ coding region of individual $\mathrm{C}$ show no evidence of additional sequence variants. The most likely explanation for these data is that the M34T variant represents a simple polymorphism that does not cause autosomal dominant hearing loss and is present in a small percentage of the general population.

Determining whether a particular DNA variant represents a disease-causing mutation or a simple polymorphism requires information about the inheritance pattern of the DNA variant within affected families and the frequency of the variant allele within the general population. Although Kelsell et al. show that M34T segregates with the profound hearing-loss phenotype in one affected family, the few individuals available for study and the presence of a second deafness-associated disorder make it difficult to draw any clear conclusions based on this family alone $e^{2,5,6}$. Kelsall et al. did not see the M34T variant in their screen of 80 chromosomes from non-related individuals, but a wider search might have revealed it.

Our results suggest that the M34T variant does not cause autosomal dominant hearing loss and emphasize the need for caution when interpreting mutation data based on a single affected family.

Daryl A. Scott, Michelle L. Kraft

Edwin M. Stone, Val C. Sheffield Richard J.H. Smith

University of Iowa Hospitals and Clinics, 200 Hawkins Drive, Iowa City,

Iowa 52242-1078, USA

e-mail: richard-smith@uiowa.edu

1. Kelsell, D. P. et al. Nature 387, 80-83 (1997).

2. Fitzgerald, D. A. \& Verbov, J. L. Br. J. Dermatol. 134, 939-942 (1996).

3. Chaib, H. et al. Hum. Mol. Genet. 3, 2219-2222 (1994).

4. Gates, G. A., Cooper, J. C. Jr, Kannel, W. B. \& Miller, N. J. Behav. Techn. Audiol. Otol. 11, 247-256 (1990).

5. Bititci, O. O. J. Laryngol. Otol. 89, 1143-1146 (1975).

6. Hatamochi, A., Nakagawa, S., Ueki, H., Miyoshi, K. \& Iuchi, I. Arch. Dermatol. 118, 605-607 (1982).

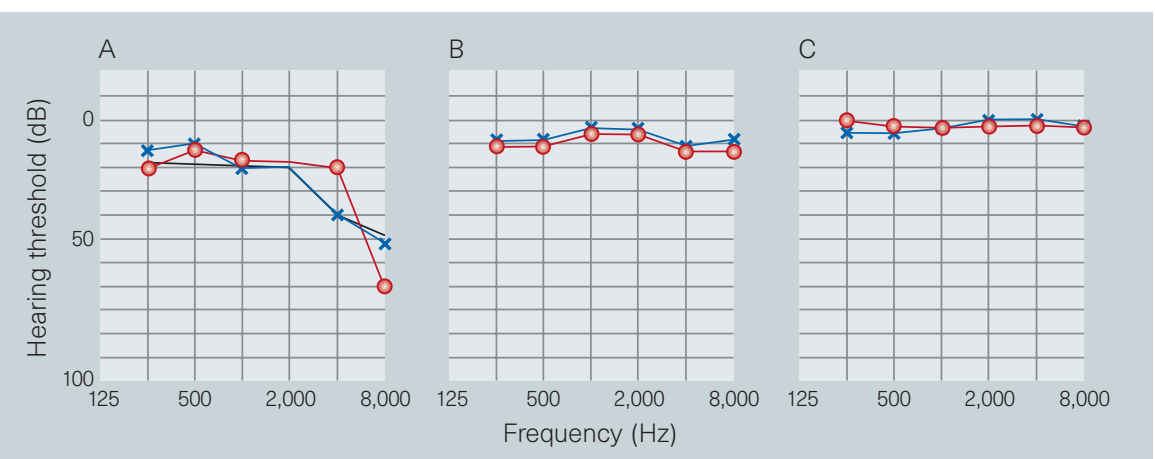

Figure 2 Pure-tone thresholds in the individuals identified in Fig. 1. Circles, right-ear thresholds; crosses, leftear thresholds. The broken line in A represents the mean pure-tone thresholds in the better ear of women aged 80-84 (ref. 4). 